\title{
Benign Malformative Lesion of the Skull: Hamartoma with Ectopic Elements or Choristoma?
}

\author{
Ece MERAM' ${ }^{1}$, Hakan KARABAĞLI ${ }^{2}$, Christine GLASTONBURY ${ }^{3}$, Tarik TiHAN' ${ }^{1}$, PInar KARABAĞLI $^{4}$ \\ Department of 'Pathology and ${ }^{3}$ Radiology, UCSF, SAN FRANCISCO, USA, \\ Department of ${ }^{2}$ Neurosurgery and ${ }^{4}$ Pathology, Selçuk University Faculty of Medicine, KONYA, TURKEY
}

\begin{abstract}
Hamartoma and choristoma are terms that describe non-neoplastic, mass-forming malformative lesions. Although each lesion has a different composition, they have been used interchangeably in many reports, especially for malformative lesions containing ectopic or heterologous elements. We report a three month-old girl who was brought to the clinic with a mass lesion at the posterior fontanel, composed of predominantly osteo-cartilaginous tissue admixed with skeletal muscle, peripheral nerve tissue, and ganglion. The mass was resected completely. The composition of this benign lesion with ectopic elements was consistent with an osteo-cartilaginous hamartoma. We discuss the biological and clinical aspects such malformative lesions within the skull to highlight the inconsistencies of the nomenclature used in the literature.
\end{abstract}

Key Words: Hamartoma, Choristoma, Ectopic tissue, Heterotopic tissue, Skull

\section{INTRODUCTION}

Malformations of the skull and surrounding tissues are a spectrum of diseases ranging from massive loss of tissue, as in anencephaly, to a mass-forming lesion such as an arteriovenous malformation. Two terms that are used to define this group of malformative lesions are hamartoma and choristoma. Hamartoma (hamartia [greek]=failure) is an overgrowth of mature tissues that normally occur in an expected area or organ, but with disorganization and often with one element predominating (1). Choristoma (choristos [greek]=separable, separated), on the other hand, is a mass of tissue histologically normal for an organ or part of the body other than the one in which it is located (1)LJ100. Choristoma is considered as a mass-forming, irregular, and heterotopic tissue (1)LJ100. Many reports use choristoma and heterotopia interchangeably (2)

Hamartomas and choristomas can occur anywhere in the body, and there have been many reports of such lesions in the skull, involving either the calvarium or the scalp, or both. Some examples include fibrous hamartoma of infancy (3), neurocristic hamartoma (4), folliculosebaceous cystic hamartoma (5), and meningothelial hamartoma (6). The lesions reported under these names have histological features compatible with the definition of hamartoma, yet many harbor heterotopic elements $(7,8)$. On the other hand, neuroglial choristomas $(2,9)$, cutaneous meningeal heterotopias (10) have been described in the skull. The latter

(Turk Patoloji Derg 2017, 33:262-267)

Received : 19.08.2014 Accepted : 29.09.2014 group of lesions is more compatible with the definition of choristoma, but the extent of heterologous elements in each may vary substantially to cast doubt on the distinction between a hamartoma and choristoma.

We present this unique case of a three-month old patient with an osteo-cartilaginous hamartoma occurring in the posterior fontanel admixed with skeletal muscle, peripheral nerve, and ganglion.

\section{CASE REPORT}

A three-month old girl was brought to the clinic when the mother noticed a bulging lesion on the posterior aspect of the skull. It was not clear how long the mass had been present. Physical examination was unremarkable except for a firm mass eroding the scalp over the posterior fontanel, with a distinct redness on the skin (Figure 1). Computed tomography of head showed a heterogeneously bright mass located at the inferior aspect of the posterior fontanel with areas having a bone-like radiodensity. T1-weighted magnetic resonance imaging demonstrated that the lesion was extra-axial, and involved the calvarium and the scalp. (Figure 2A-E). Intraoperatively, the mass was found to be located within the posterior fontanel and adjacent to superior sagittal sinus and the right parietal bone. The lesion had eroded the skull locally, reaching the subcutaneous tissue of the scalp. Inferiorly, the mass was attached to the dura mater but did not penetrate this structure. A grosstotal resection was achieved without disrupting the dura.

Correspondence: Tarik TiHAN

UCSF, Department of Pathology, SAN FRANCISCO, USA

E-mail: tarik.tihan@ucsf.edu Phone: +01 4155149332 
Excised mass consisted of a single, irregular, firm bony and fibrous tissue measuring $2.5 \times 2.3 \times 1.5 \mathrm{~cm}$. Serial sections of the mass demonstrated an admixture of dense, pearly white cartilage tissue with bone and dark brown bone marrow elements arranged in a haphazard fashion (Figure 3).

The patient returned for a clinical visit 7 months following the surgical procedure and radiological and clinical studies demonstrated no evidence of disease.

\section{Histopathological Findings}

On histological sections, the lesion was well-circumscribed and composed predominantly of osseous tissue with mature bone marrow elements (Figure 4A). Bone marrow was hyperplastic and showed tri-lineage hematopoiesis. In addition, there were foci of peripheral nerve (Figure 4B), ganglion (Figure 4D), and mature cartilage within the mass. The peripheral nerve and ganglion tissues were present within the osseous component, trapped among cartilaginous and osseous elements. The lesion also contained skeletal muscle. In addition, there was immature adipose tissue with dense fibrous septa at the periphery, which was considered to be normal subcutaneous adipose tissue (Figure 4E). The osseous component had wellformed, unremarkable bony lamellae as well as abnormal, woven bone and foci of irregular maturation zones. Both the peripheral nerve tissue and the ganglion within the osseous component demonstrated strong positive staining with the antibodies against S-100 protein and Glial fibrillary acidic protein (GFAP) (Figure 4B-C). No glial elements were identified in the lesion using GFAP and Olig-2 stains. An immunohistochemical stain for Ki-67 (MIB1) showed no positive cells within the osseous, peripheral nerve or ganglion tissue, but there were abundant positive nuclei within the bone marrow.

\section{DISCUSSION}

Presence of certain features in the aforementioned lesion, such as mature tissue from two germ layers, ectopic neuronal elements and overgrowth of indigenous tissues were important in identifying this mass-forming skull lesion as a malformative process. Thus, the terms teratoma, choristoma or hamartoma were considered in the diagnosis of this lesion.

The lesion showed elements arising from two different germ layers (mesodermal and ectodermal), and it did not have any immature components. The possibility of a benign mature teratoma was entertained since there are ample reports of benign mature teratomas or the so-called dermoid cysts, found along the suture lines of cranial bones (11). Mature teratomas may show no or low mitotic index and are mass forming lesions, and may demonstrate heterologous elements such as skeletal muscle, cartilage or neural tissue. In addition, some cranial teratomas can have mature bone and bone marrow. In case the term teratoma is used to characterize a hamartomatous lesion, it may be possible to consider this lesion in this category. However, the skull based teratomas or dermoid cysts are predominantly composed of epidermal, dermal and adnexal structures along with keratin, and they are not predominantly composed of osteo-cartilaginous elements. Thus, our case is not a dermoid cyst. Recent studies also suggested that some lesions previously reported as teratomas are non-clonal lesions, and are better classified as hamartoma (12). If we consider the usage of the term "teratoma" to represent a neoplastic growth, then our lesion would not be considered a teratoma.

Choristoma could be considered in the differential diagnosis of this lesion because of the presence of ectopic or heterotopic non-neoplastic tissues including skeletal muscle, peripheral nerve and ganglion. One does not expect to identify skeletal muscle in this location even though the tissue may be a remnant from the stage of skeletal muscle development and migration in the lower occipital region. The peripheral nerve tissue and ganglion were presumed to be heterotopic tissue since they were within the mass lesion amidst the osseous and cartilaginous elements, and not in the surrounding soft tissue. However, the peripheral nervous tissue could be considered not ectopic in this region, since

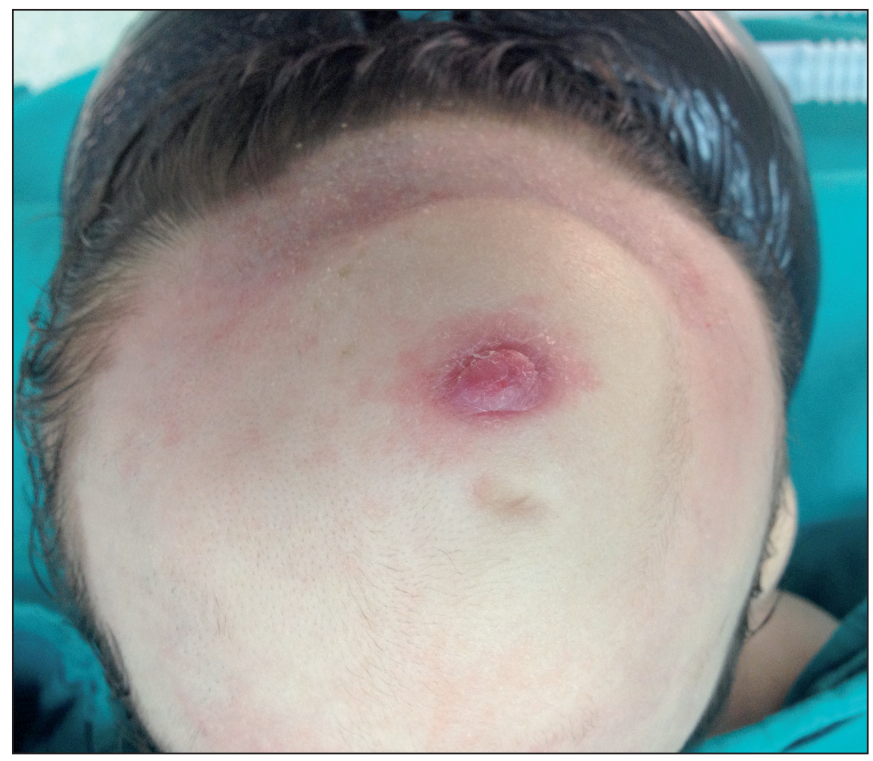

Figure 1: Preoperative external view of the lesion showed erythema and erosion of the scalp as well as swelling at the expected location of the occipital fontanel. 

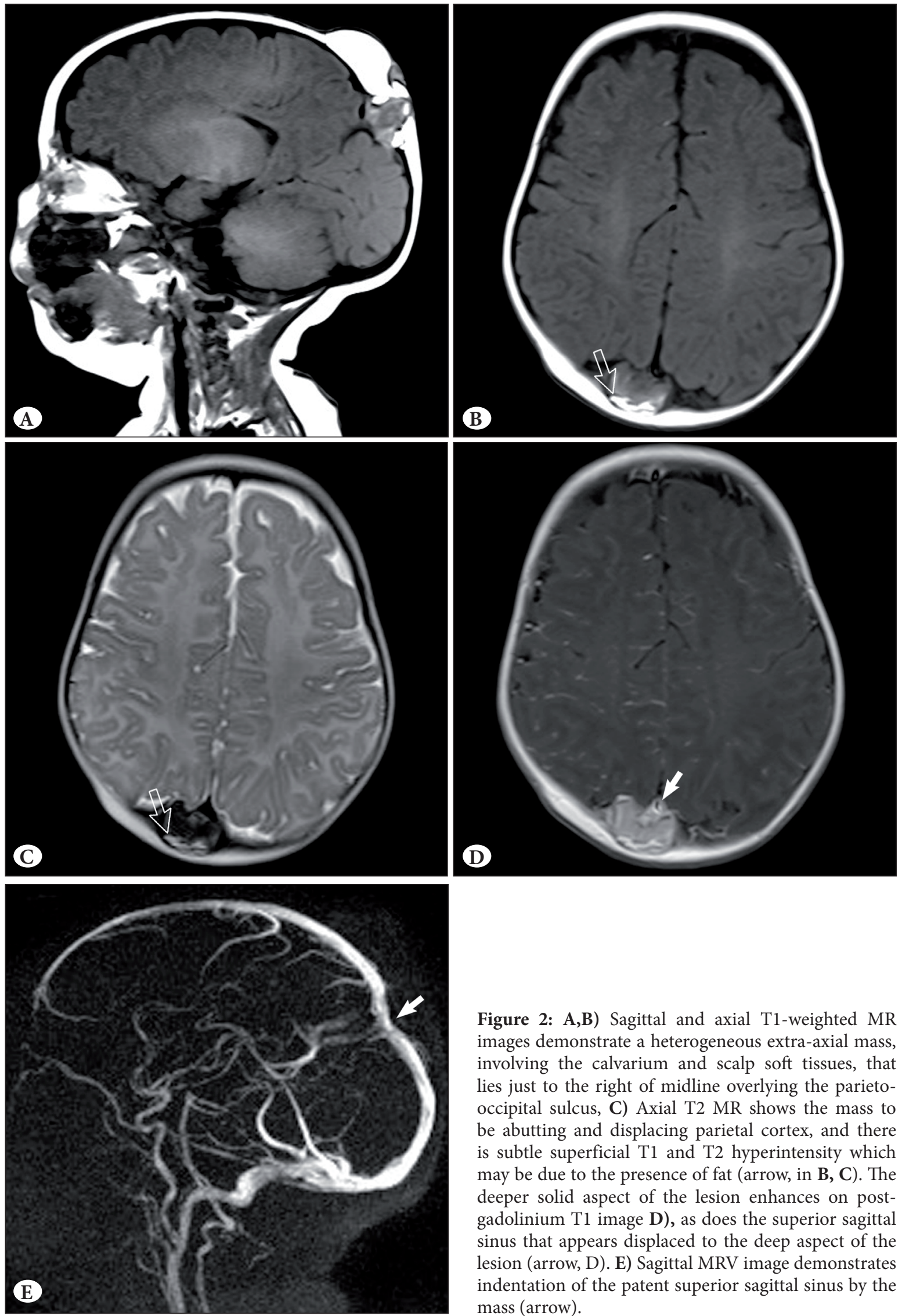

Figure 2: A,B) Sagittal and axial T1-weighted MR images demonstrate a heterogeneous extra-axial mass, involving the calvarium and scalp soft tissues, that lies just to the right of midline overlying the parietooccipital sulcus, C) Axial T2 MR shows the mass to be abutting and displacing parietal cortex, and there is subtle superficial T1 and T2 hyperintensity which may be due to the presence of fat (arrow, in B, C). The deeper solid aspect of the lesion enhances on postgadolinium T1 image D), as does the superior sagittal sinus that appears displaced to the deep aspect of the lesion (arrow, D). E) Sagittal MRV image demonstrates indentation of the patent superior sagittal sinus by the mass (arrow). 


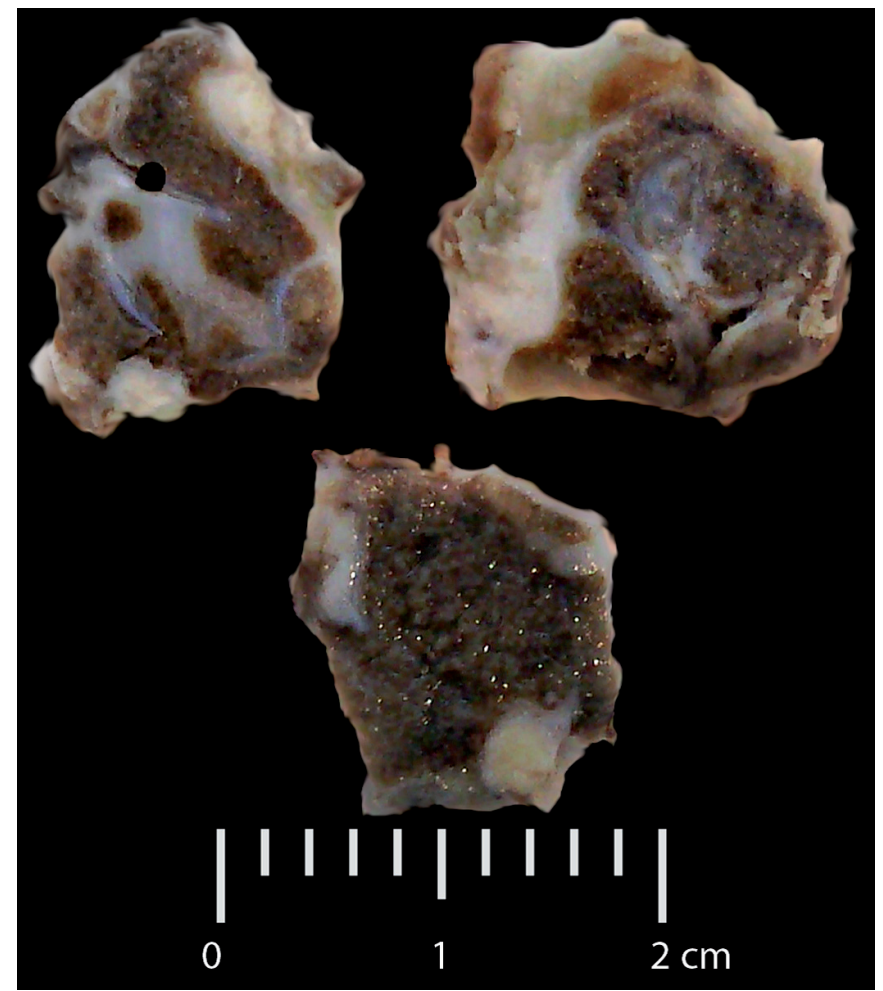

Figure 3: Macroscopic appearance of the surgical resection material sectioned along the long axis demonstrated predominantly bone and bone marrow admixed with dense, white cartilaginous tissue arranged in a haphazard fashion.

peripheral nerves can be found in the scalp, dura mater or the periosteum. The ganglion found in this lesion should be considered truly ectopic. The closest extra-axial ganglion tissue to the lesion would be the base of the skull. Therefore, presence of this heterotopic tissue raises the possibility of a choristoma. Since aberrant rest of cells can be seen due to directional disturbance of precursor tissue migration rather than from implantation of foreign cells. Yet, this process is still considered a choristoma. Differentiation of neural crest cells and their development to bone, cartilage, muscle, nerve and ganglion in the head and neck region $(13,14)$, make it plausible to think that such a lesion can be formed through separation of a group of neural crest cells during their migration. Therefore, misplacement of cells to be differentiated into various tissues can be the underlying the pathophysiology of such a malformative lesion.

Despite the presence of small rests of ectopic tissues, the overwhelming majority of the mass was mature bone, cartilage and marrow that are native to the site. In addition, the disorganized but mature tissue composition and nonneoplastic histological features favors a hamartoma. Even though some lesions in the oropharyngeal region were reported as "osteo-cartilaginous choristoma" $(15,16)$, the greatest portion of the abnormal tissue was not ectopic to the site, and favored a predominantly hamartomatous process with smaller ectopic elements. In contrast to this report, Suster and Rosai have reported a lesion that they defined as a scalp hamartoma with ectopic meningothelial elements (7).

The uncertainty on the variation in the concepts and their usage in the literature may be confusing, yet the lesion in our patient was classified as a hamartoma since it was predominantly a disorganized overgrowth of native osteocartilaginous tissue. However, one could name this lesion a "choristoma", since heterotopic elements were found in the lesion. In addition, many studies in the literature have failed to provide a distinction between a choristoma and a hamartoma with ectopia, and it is not really clear whether there are any. This uncertainty results in the situation that the same lesion could be reported as hamartoma by some, choristoma by others, and yet, as both in other studies (17). In fact, benign Triton tumors are often named as both neuromuscular hamartoma and neuromuscular choristoma in the same text (18). In other studies, it is possible to see the lesion described as a hamartoma (19), or choristoma (20), despite the same histological appearance.

There is little doubt that the classification of a particular malformative lesion can change based on the authors' interpretation of the terminology. While the natural biology of both lesions are benign, and the name does not significantly impact the patient's outcome, the usage of these terms interchangeably and without concern to their actual composition creates a diagnostic and classification problem. Yet, the distinction of these two terms might be significant in search of their underlying pathophysiology as well as in recognizing syndromic patients in whom lesions have originally been described as either one or other. This confusion of terminology can also cause patients to second-guess the accuracy and the reliability of pathological diagnoses. Finally, regardless of the nature of the lesions, accurate and scientific classification of diseases is paramount to discovery of disease mechanisms.

The correct classification of a malformative lesion becomes even more indecisive in the presence of heterotopic element within what is essentially a hamartomatous formation. We are not quite certain that the extent of heterologous elements in a particular malformative lesion would change the nature or the name of the pathological process. Thus, it may be worthwhile to consider what is meant by hamartoma with ectopic/heterotopic elements versus a mere choristoma. Our case report of a predominantly osteo-cartilaginous mass with skeletal muscle, peripheral nerve and ganglion is 

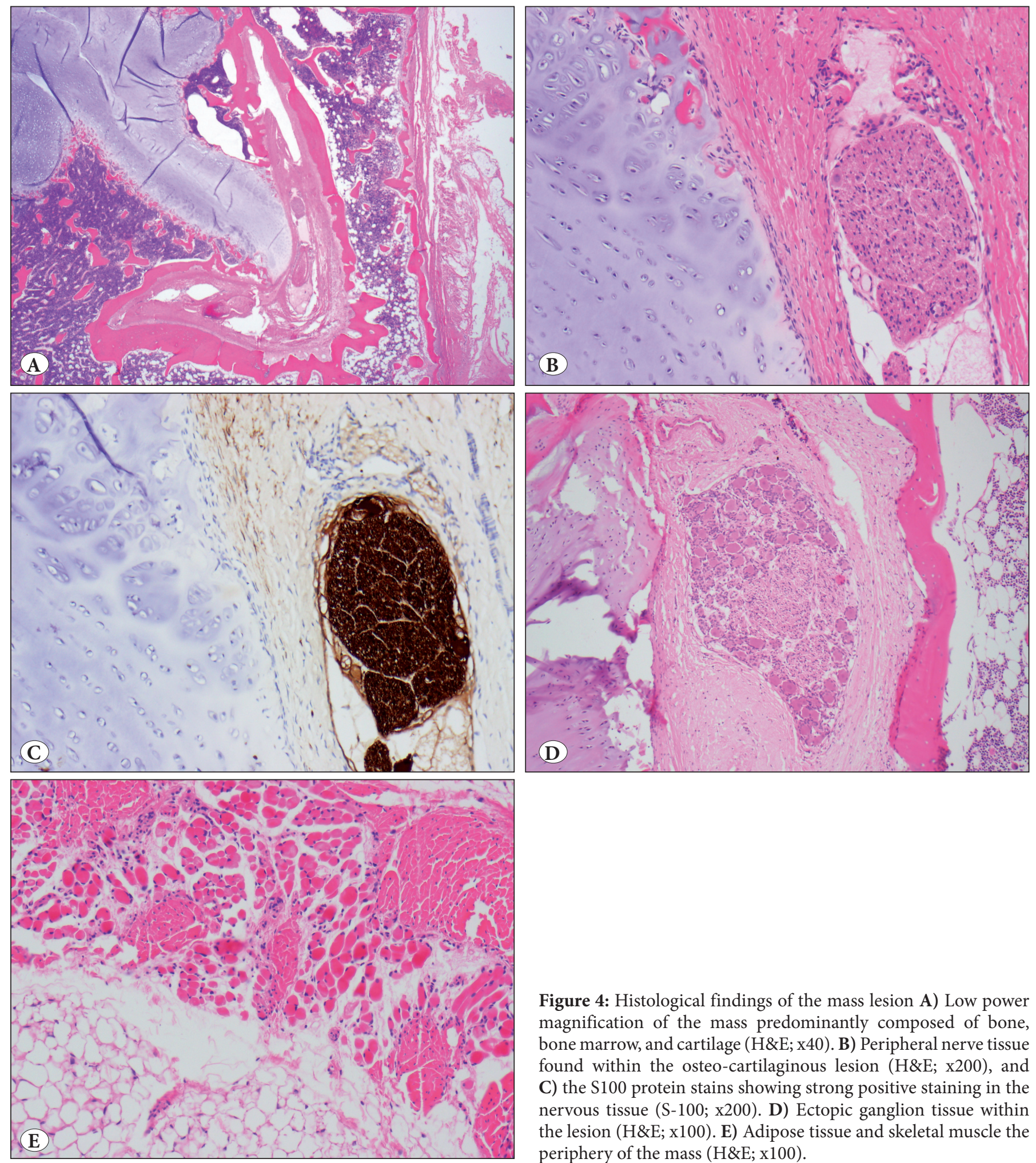

Figure 4: Histological findings of the mass lesion A) Low power magnification of the mass predominantly composed of bone, bone marrow, and cartilage (H\&E; x40). B) Peripheral nerve tissue found within the osteo-cartilaginous lesion (H\&E; x200), and C) the $S 100$ protein stains showing strong positive staining in the nervous tissue (S-100; x200). D) Ectopic ganglion tissue within the lesion (H\&E; x100). E) Adipose tissue and skeletal muscle the periphery of the mass (H\&E; $\mathrm{x} 100)$.

aimed at demonstrating the difficulties in classification and nomenclature of these benign lesions, most probably due to the fact that it has little, if any impact in patients' prognoses. Yet such simplification in the usage of terminology and confusion of terms can render the diagnosis suspect, overlook a particular mechanism or etiology, and make the study of such lesions quite cumbersome. Better recognition of these pathological processes, their pathogenesis, and 
accurate definition and use of terminology should help clarify the confusion in the literature.

In conclusion, hamartoma and choristoma are commonly used terms in medicine, yet certain lesions may be difficult to define precisely as either one. Accordingly, categorization of lesions as either choristoma or hamartoma is not always straightforward, and some have even used these terms interchangeably. Furthermore, it is not clear whether a lesion should be called a hamartoma if it includes ectopic (or heterotopic) tissue, since ectopic tissue has been typically associated with the definition of choristoma. We present this ambiguity in literature and a case of an osteocartilaginous hamartoma in the skull of a three month-old girl with ectopic elements of skeletal muscle, peripheral nerve and ganglion.

\section{REFERENCES}

1. Dorland WAN: Dorland's illustrated medical dictionary. Philadelphia, W.B. Saunders Co. v.

2. Dunham E, Armeni M. Glial choristoma of the temporal bone in a 7-month-old infant. JAMA Otolaryngol Head Neck Surg. 2013;139:944-6.

3. Mitchell ML, di Sant'Agnese PA, Gerber JE. Fibrous hamartoma of infancy. Hum Pathol. 1982;13:586-8.

4. Smith KJ, Mezebish D, Williams J, Elgart ML, Skelton HG. The spectrum of neurocristic cutaneous hamartoma: Clinicopathologic and immunohistochemical study of three cases. Ann Diagn Pathol. 1998;2:213-23.

5. Singh N, Chandrashekar L, Thappa DM, Kar R. Solitary papule over scalp. Dermatol Online J. 2014;20:22334.

6. Hirakawa E, Kobayashi S, Terasaka K, Ogino T, Terai Y, Ohmori M. Meningeal hamartoma of the scalp. A variant of primary cutaneous meningioma. Acta Pathol Jpn. 1992;42:353-7.

7. Suster S, Rosai J. Hamartoma of the scalp with ectopic meningothelial elements. A distinctive benign soft tissue lesion that may simulate angiosarcoma. Am J Surg Pathol. 1990;14:1-11.
8. Ferran M, Tribo MJ, Gonzalez-Rivero MA, Alameda F, Pujol RM. Congenital hamartoma of the scalp with meningothelial, sebaceus, muscular, and immature glandular components. Am J Dermatopathol. 2007;29:568-72.

9. Hirano S, Houdou S, Hasegawa M, Kamei A, Takashima S. Clinicopathologic studies on leptomeningeal glioneuronal heterotopia in congenital anomalies. Pediatr Neurol. 1992;8:4414.

10. Battistella M, Guedj N, Fallet-Bianco C, Bodemer C, Brousse N, Fraitag $\mathrm{S}$. The histopathological spectrum of cutaneous meningeal heterotopias: Clues and pitfalls. Histopathology. 2011;59:407-20.

11. Ruge JR, Tomita T, Naidich TP, Hahn YS, McLone DG. Scalp and calvarial masses of infants and children. Neurosurgery. 1988;22:1037-42.

12. Sinnock KL, Perez-Atayde AR, Boynton KA, Mutter GL. Clonal analysis of sacrococcygeal "teratomas". Pediatr Pathol Lab Med. 1996;16:865-75.

13. Mishina Y, Snider TN. Neural crest cell signaling pathways critical to cranial bone development and pathology. Exp Cell Res. 2014;325:138-47.

14. Gong SG. Cranial neural crest: Migratory cell behavior and regulatory networks. Exp Cell Res. 2014;325:90-5.

15. Roper A, Subar D, Benatar B, Kobbe AE. Osteocartilaginous choristoma: A case report. J Laryngol Otol. 2006;120:E23.

16. Wesley RK, Zielinski RJ. Osteocartilaginous choristoma of the tongue: Clinical and histopathologic considerations. J Oral Surg. 1978;36:59-61.

17. Tobias S, Kim CH, Sade B, Staugaitis SM, Lee JH. Neuromuscular hamartoma of the trigeminal nerve in an adult. Acta Neurochir (Wien). 2006;148:83-7; discussion 87.

18. Goldblum JR, Folpe AL, Weiss SW, Enzinger FM, Weiss SW. Enzinger and Weiss's soft tissue tumors. 6th ed. Philadelphia, PA: Saunders/Elsevier; 2014.

19. Castro DE, Raghuram K, Phillips CD. Benign triton tumor of the trigeminal nerve. AJNR Am J Neuroradiol. 2005;26:967-9.

20. Thakrar R, Robson CD, Vargas SO, Meara JG, Rahbar R, Smith ER. Benign triton tumor: Multidisciplinary approach to diagnosis and treatment. Pediatr Dev Pathol. 2014;17:400-5. 